\title{
Hadjar, Andreas
}

\section{Erziehungswissenschaft(en) in Luxemburg. Eine feste Säule in der noch jungen luxemburgischen Hochschullandschaft \\ Erziehungswissenschaft 27 (2016) 52, S. 41-53}

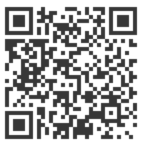

Quellenangabe/ Reference:

Hadjar, Andreas: Erziehungswissenschaft(en) in Luxemburg. Eine feste Säule in der noch jungen luxemburgischen Hochschullandschaft - In: Erziehungswissenschaft 27 (2016) 52, S. 41-53 - URN: urn:nbn:de:0111-pedocs-122244 - DOI: 10.25656/01:12224

https://nbn-resolving.org/urn:nbn:de:0111-pedocs-122244

https://doi.org/10.25656/01:12224

in Kooperation mit / in cooperation with:

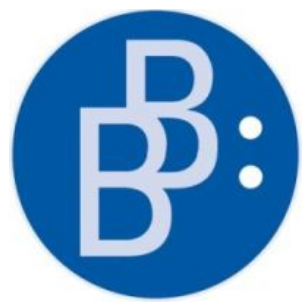

https://www.budrich.de

\section{Nutzungsbedingungen}

Gewährt wird ein nicht exklusives, nicht übertragbares, persönliches und beschränktes Recht auf Nutzung dieses Dokuments. Dieses Dokument ist ausschließlich für den persönlichen, nicht-kommerziellen Gebrauch bestimmt. Die Nutzung stellt keine Übertragung des Eigentumsrechts an diesem Dokument dar und gilt vorbehaltlich der folgenden Einschränkungen: Auf sämtlichen Kopien dieses Dokuments müssen alle Urheberrechtshinweise und sonstigen Hinweise auf gesetzlichen Schutz beibehalten werden. Sie dürfen dieses Dokument nicht in irgendeiner Weise abändern, noch dürfen Sie dieses Dokument für öffentliche oder kommerzielle Zwecke vervielfältigen, öffentlich ausstellen, aufführen, vertreiben oder anderweitig nutzen.

Mit der Verwendung dieses Dokuments erkennen Sie die Nutzungsbedingungen an.

$$
\text { Dokuments erkennen Sie die }
$$

\section{Terms of use}

We grant a non-exclusive, non-transferable, individual and limited right to using this document.

This document is solely intended for your personal, non-commercial use. Use of this document does not include any transfer of property rights and it is conditional to the following limitations: All of the copies of this documents must retain all copyright information and other information regarding legal protection. You are not allowed to alter this document in any way, to copy it for public or commercial purposes, to exhibit the document in public, to perform, distribute or otherwise use the document in public.

By using this particular document, you accept the above-stated conditions of use.

\section{Kontakt / Contact:}

\section{peDOCS}

DIPF | Leibniz-Institut für Bildungsforschung und Bildungsinformation Informationszentrum (IZ) Bildung

E-Mail: pedocs@dipf.de

Internet: www.pedocs.de

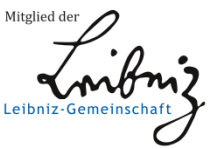




\section{INHALTSVERZEICHNIS}

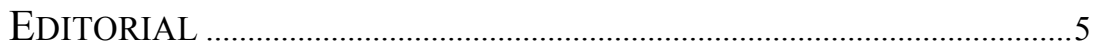

BEITRÄGE ZUM THEMA ,ERZIEHUNGSWISSENSCHAFT IN DEN NACHBARLÄNDERN"

Jean-Luc Patry \& Josef Thonhauser

Erziehungs- und bildungswissenschaftliche Forschung in Österreich .7

Hubert Ertl

Die Erziehungswissenschaft im Vereinigten Königreich: Die erziehungswissenschaftliche Forschungslandschaft im Spiegel der Forschungsevaluation.

Andreas Hadjar

Erziehungswissenschaft(en) in Luxemburg - Eine feste Säule in der noch jungen luxemburgischen Hochschullandschaft

Theo Wubbels

Educational Sciences in the Netherlands: State of Affairs and

Challenges 55

Lucien Criblez

Erziehungswissenschaft in der Schweiz - aktuelle Situation und Entwicklungstendenzen

\section{LAUDATIONES}

\section{Hans-Christoph Koller}

Laudatio für Prof. Dr. Heinz-Elmar Tenorth anlässlich der Verleihung des Ernst-Christian-Trapp-Preises.

Ingrid Miethe, Hannelore Faulstich-Wieland \& Dietrich Benner

Laudatio für Prof. Dr. Sigrid Blömeke anlässlich der Verleihung des

DGfE Forschungspreises

Malte Brinkmann

Laudatio für Dr. Thomas Rucker anlässlich der Verleihung des

Förderpreises der DGfE

Stefanie Hartz \& Ivo Züchner

Laudatio für Dr. Silvia Salchegger anlässlich der Verleihung des

Förderpreises der DGfE 


\section{Marcelo Caruso}

Laudatio für Prof. Dr. Ingrid Gogolin anlässlich der Verleihung der Ehrenmitgliedschaft der DGfE

Marcelo Caruso

Laudatio für Prof. Dr. Klaus-Jürgen Tillmann anlässlich der Verleihung der Ehrenmitgliedschaft der DGfE.

Marcelo Caruso

Laudatio Horst Weishaupt anlässlich der Verleihung der

Ehrenmitgliedschaft der DGfE 105

Autorinnen und Autoren der Laudationes 106

\section{MitTEILUNGEN DES VORSTANDS}

Rechenschaftsbericht über die Vorstandsperiode März 2014 bis März

2016 an die Mitgliederversammlung in Kassel am 15. März 2016

17. Kolloquium „Forschungsberatung“ der DGfE

\section{BERICHTE AUS DEN SEKTIONEN}

Sektion 1 - Historische Bildungsforschung ................................................... 125

Sektion 2 - Allgemeine Erziehungswissenschaft ........................................... 130

Sektion 4 - Empirische Bildungsforschung ................................................ 134

Sektion 8 - Sozialpädagogik und Pädagogik der frühen Kindheit................. 135

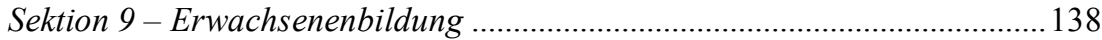

NOTIZEN

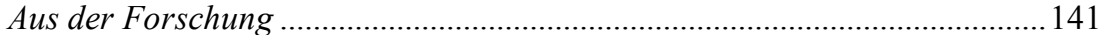

Aus Wissenschaft und Lehre ......................................................................... 147

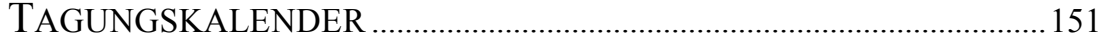

PERSONALIA

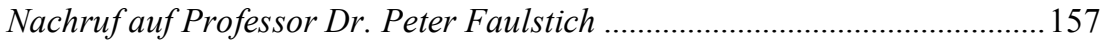

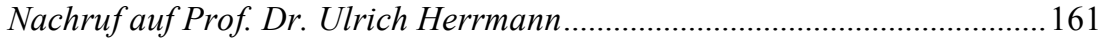

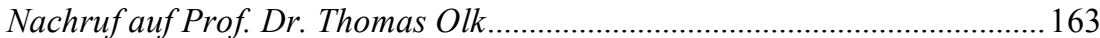




\title{
Erziehungswissenschaft(en) in Luxemburg - Eine feste Säule in der noch jungen luxemburgischen Hochschullandschaft
}

\author{
Andreas Hadjar
}

\section{Einleitung}

Die Heimat der Erziehungswissenschaft(en) in Luxemburg ist die junge Forschungsuniversität des kleinen Großherzogtums, die 2003 gegründet wurde. Auch wenn aufgrund der interdisziplinären Strukturierung der Fakultäten und Zentren der Universität Luxemburg kein „Institut für Erziehungswissenschaft" existiert, gibt es doch ganz verschiedene Einheiten - Forschungseinheiten und Institute - in denen die Disziplin der Erziehungswissenschaft(en) eine zentrale Rolle spielt. Auf der Ebene akademischer und berufsorientierter Studiengänge (Bachelor- und Masterstudiengänge, Doktorandenausbildung, Weiterbildungsstudiengänge) gibt es explizite erziehungswissenschaftliche Angebote. Außerhalb der Universität gibt es noch einen weiteren Träger der Erziehungswissenschaft: das Lehrer/innenbildungs- und Weiterbildungsinstitut Institut de formation de l'Education nationale (IFEN), das dem luxemburgischen Bildungsministerium direkt untersteht. Zudem ist auch das der oberen Sekundarstufe zuzuordnende Lycée technique pour professions éducatives et sociales (LTPES) zu erwähnen, das bis 2003 das Ausbildungsinstitut für Erzieherinnen und Erzieher in Luxemburg war und somit auch eine erziehungswissenschaftliche Tradition hat.

Der folgende Länderbericht wird sich auf die Erziehungswissenschaft in Forschung und Lehre an der Universität Luxemburg konzentrieren und außeruniversitäre Verortungen nur sehr am Rande zu beleuchten. Die Universität ist 2003 aus dem Centre Universitaire de Luxembourg (CUNLUX), einer tertiären Forschungs- und Lehreinrichtung auf (Fach-)Hochschulniveau, hervorgegangen. Die Ausbildung von Lehrerinnen und Lehrern wurde vom Institut Supérieur d'Études et de Recherches Pédagogiques (ISERP) getragen (Meyer 2008). Während die Grundschullehrerinnen und Grundschullehrer ihre gesamte Ausbildung in Luxemburg an dem entsprechenden Institut verbleiben konnten, studierten die Sekundarlehrerinnen und Sekundarlehrer im Ausland, um dann nach dem ausländischen Studienabschluss ihres Faches ein vom ISERP - auch mit erziehungswissenschaftlichen Ausbildungsanteilen begleitetes Referendariat in Luxemburg zu absolvieren. Die Grundprinzipien der noch jungen Universität Luxemburg sind Mehrsprachigkeit, Interdisziplinarität und Internationalisierung (Powell 2015). Mit der Mehrsprachigkeit wird der Tatsache Rechnung getragen, dass in Luxemburg offiziell drei 
Amtssprachen genutzt werden: Lëtzebuergisch, Deutsch und Französisch. Für die junge (Forschungs-)Universität Luxemburg mit einer starken internationalen Ausrichtung ist zudem Englisch von zentraler Bedeutung, das neben Deutsch und Französisch zu den Universitätssprachen gehört. Es gibt mono-, bi- und multilinguale Studiengänge, weshalb Angestellte mindestens zwei Universitätssprachen sicher beherrschen sollen. Die Internationalität der Universität zeigt sich u.a. in weltweiten Kooperationen in Forschung und Lehre bzw. einer starken Unterstützung von internationalem Austausch - etwa von internationalen Tagungen und Projekten sowie Studierenden-, Akademikerinnen- und Akademiker-Mobilität. So müssen Bachelorstudierende mindestens ein Semester im Ausland verbringen. Die Forschenden, Dozierenden und Studierenden kommen aus ganz verschiedenen Regionen der Welt - die 1506 Mitarbeitenden (u.a. 238 Professorinnen, Professoren und Dozierende, 416 Doktoranden mit Mitarbeiterstatus) aus 25 Ländern, die 6287 Studierenden aus 110 Ländern (Universität Luxemburg 2016). Die Gründer der Universität verfolgten das Modell einer interdisziplinären Forschungsuniversität, die weniger an Disziplinen und stärker an Forschungsfeldern orientiert ist. Die drei Fakultäten - a) Fakultät für Naturwissenschaften, Technologie und Kommunikation, b) Fakultät für Rechts-, Wirtschafts- und Finanzwissenschaften und c) Fakultät für Sprachwissenschaften und Literatur, Geisteswissenschaften, Kunst und Erziehungswissenschaften (FLSHASE) - sind entsprechend in Forschungseinheiten untergliedert, welche wiederum in Institute gegliedert sind. Wie bereits in der Auflistung der vollständigen Namen der Fakultäten ersichtlich, sind die Erziehungswissenschaften in der letztgenannten Fakultät FLSHASE verortet. Diese ist die größte der drei Fakultäten und beheimatet 400 Lehrende und Forschende sowie 2500 Studierende. Die in verschiedenen multidiziplinären Forschungseinheiten, Instituten und Studienprogrammen vertretenen Disziplinen sind Erziehungswissenschaft(en), Sozialwissenschaften (u.a. Soziologie, Politikwissenschaft, Soziale Arbeit), Kulturwissenschaft, Sprachwissenschaften und Literaturwissenschaften, Psychologie, Philosophie, Geschichte und Geographie.

Im nächsten Abschnitt werden die mannigfaltigen Forschungsstrukturen an der Universität Luxemburg mit einem erziehungswissenschaftlichen Schwerpunkt vorgestellt und die Publikationskultur anhand von Zahlen verdeutlicht. Im dritten Abschnitt die Personalstruktur - und damit die Basis für den Output an Forschung, Publikationen und Lehre - beschrieben. Im vierten Abschnitt werden die verschiedenen Studiengänge mit erziehungswissenschaftlichem Bezug vorgestellt, um in einem fünften Abschnitt Zahlen zu den Studierenden an der Universität Luxemburg sowie spezifisch in den erziehungswissenschaftlichen Studiengängen zu präsentieren. 


\section{Forschungs- und Publikationskulturen}

\subsection{Forschungseinheiten, Institute, An-Institut und Forschungspriorität}

Die Fakultät FLSHASE ist in drei Forschungseinheiten untergliedert, die wiederum in 17 kleinere Einheiten - Institute - unterteilt sind. Wie bereits erwähnt finden sich die Erziehungswissenschaft(en) in verschiedenen Strukturen. Als Heimat der Erziehungswissenschaft kann die multdisziplinären Forschungseinheit Education, Culture, Cognition \& Society (ECCS) bezeichnet werden, der 160 Forschende aus den Erziehungswissenschaft(en), der Geschichtswissenschaft, der Linguistik, der Neurowissenschaft, der Psychologie, der Soziologie, der Anthropologie und der Informatik angehören. Bildung und Erziehung sind die Kernthemen. Das Forschungsprofil umfasst Lehrplan- und Policy-Studien, Lehrerinnen- und Lehrerbildung, kognitive Entwicklung, Lernprozesse, Ungleichheitsforschung, Migration und Multilingualismus in der Schule und in der höheren Bildung.

Die Forschungseinheit ECCS ist in sechs (Forschungs-)Institute untergliedert, die ebenso einen multidisziplinären Ansatz haben. Das Institute for Applied Educational Sciences (AES) beschäftigt sich mit Lehr-Lern-Prozessen in verschiedensten Kontexten (Klassenzimmer, außerunterrichtliche Schulaktivitäten, Elternhaus etc.) mit dem Ziel, die Qualität des Unterrichts und von Bildung allgemein zu verbessern. Entsprechend liegen die Schwerpunkte der qualitativen und quantitativen Untersuchungen im Bereich der Schul- und Unterrichtsforschung sowie der Didaktik. Das Institute for Cognitive Science and Assessment (COSA) vereint ebenso das Wissen verschiedener disziplinärer Ansätze (Psychologie, Kognitionswissenschaft, Psychometrie). Zu den Forschungsschwerpunkten gehören das computer-basierte Assessment (etwa von Schülerinnen und Schülern), kognitive Neurowissenschaft, MenschComputer-Interaktion und Activity Research. Das Institute of Education and Society versammelt gesellschafts- und sozialwissenschaftliche Perspektiven auf Bildung und Bildungsprozesse (Bildungstheorie, -philosophie, -geschichte, -soziologie sowie Kulturwissenschaften). Das Hauptinteresse gilt Bildungsprozessen und Bildungssystemen unter Berücksichtigung kultureller, politischer und ökonomischer Kontexte. Eine besondere Bedeutung kommt dabei einer nationenübergreifenden und einer international- und historischvergleichenden Perspektive zu. Gegenstände für quantitative und qualitative, historische und institutionenfokussierte Analysen sind Bildungspolitiken und die Entwicklung von Bildungssystemen und Institutionen sowie Erziehungsund Bildungsprozesse, Lehren und Lernen in und außerhalb von Bildungsins-

1 Für weitere Informationen zur Forschungseinheit und den Instituten: http://wwwde.uni.lu/r echerche/flshase/education_culture_cognition_and_society_eccs. 
titutionen. Das Institute of Lifelong Learning and Guidance (LLLG) untersucht vor allem Lernprozesse außerhalb der Schule und nimmt aus einer psychologischen Perspektive heraus kognitive Anpassungs- und Lernstrategien an bedeutsamen Übergängen im Lebensverlauf in den Blick. Dazu gehören Übergänge von der Schule in den Beruf, innerhalb von professionellen Domänen, aus dem Arbeitsleben in das Rentenalter sowie von Erwerbstätigkeit in die Arbeitslosigkeit und umgekehrt. Das Institute for Research on Multilingualism (Mling) fokussiert einerseits auf Mehrsprachigkeit in Schule, Beruf, Hochschulbildung und Gesellschaft, andererseits auf die Sprachaneignung und die kognitive Entwicklung in multilingualen formalen und informellen Umwelten von der Kindheit bis ins Erwachsenenalter. Die disziplinäre Vielfalt im Institut inkludiert formale und kognitive Linguistik, Psychologie, Sprachdidaktik und Sozialsemiotik. Das Institute of Teacher Professionalization and Psychology of Education (TPPE) hat einen genuinen pädagogisch-psychologischen Schwerpunkt. Lehrerinnen- und Lehrerbildung und professionelle Entwicklung, Biographien von Lehrerinnen und Lehrern, die psychologische und bildungsbezogene Messung im Hinblick auf pädagogische Diagnostik und Bewertung und allgemeine psychologische Grundlagen von Bildungsprozessen gehören zu den Forschungsschwerpunkten. Damit rücken Lehrstile, pädagogische Diagnostik und der Lehrberuf in das Zentrum quantitativer und qualitativer Forschung.

Im Hinblick auf erziehungswissenschaftliche Forschung ist ein Research and Transfer Centre - in der deutschen Wissenschaftslandschaft mit einem An-Institut vergleichbar - zu nennen, das der Fakultät für Sprachwissenschaften und Literatur, Geisteswissenschaften, Kunst und Erziehungswissenschaft(en) zugeordnet ist. Das Luxembourg Centre for Educational Testing (LUCET) dient der Erforschung und dem Transfer wissenschaftlicher Befunde auf dem Gebiet der Bewertungs- und Messinstrumente im Bildungsbereich. $\mathrm{Zu}$ den Auftragsforschungen gehört neben den Luxemburgischen PISA-Studien auch ein Bildungsmonitoring. Ziel ist die Erstellung einer längsschnittlichen Datenbasis zur Entwicklung der Kompetenzen von Schülerinnen und Schülern, ihren Einstellungen und ihrer Motivation, die für evidenzbasierte Bildungspolitik und Schulentwicklung herangezogen werden kann. Ein weiterer bedeutsamer Bereich betrifft die Entwicklung von Messinstrumenten zur Kompetenzmessung, die insbesondere für den multikulturellen und multilingualen luxemburgischen Kontext geeignet sind. Der disziplinäre Hintergrund des Instituts wird als genuin erziehungswissenschaftlich und pädagogisch-psychologisch beschrieben.

Schließlich gibt es an der Universität Luxemburg ein einheitsübergreifendes strukturelles Instrument zur Forschungsförderung: Forschungsprioritäten. Mit diesen Strukturen, die alle vier Jahren im Rahmen der Erstellung der in Luxemburg üblichen Vier-Jahres-Pläne inhaltlich und strukturell ausgestaltet werden, sind finanzielle Unterstützungen verbunden, die in Personal, Tagun- 
gen und Vorträge, Publikationen und kleinere Forschungen investiert werden können. Die für die Erziehungswissenschaft(en) relevante Forschungspriorität ist die Research Priority: Education, welche schon im vorhergehenden Vier-Jahresplan berücksichtigt wurde und in der aktuellen Periode 2014 bis 2017 einen Fokus auf Mehrsprachigkeit und Diversität hat. Themen betreffen den Spracherwerb und das allgemeine schulische Lernen in mehrsprachigen Gesellschaften und entsprechenden Bildungssystemen, den Umgang mit Sprachenvielfalt, aber auch generelle ungleichheitsbezogene Fragestellungen wie die Benachteiligungen entlang bestimmter Merkmale wie sozioökonomischer Hintergrund, Migrationshintergrund und sprachlicher Hintergrund sowie Fragen von Förderbedarfen und schulischer Inklusion. Vertreten sind wiederum eine Reihe von Disziplinen: Erziehungswissenschaft(en) und Didaktik, Linguistik, Psychologie, Kognition und Neurowissenschaft, Geschichte, Soziologie und Anthropologie.

\subsection{Publikationen}

Die Universität Luxemburg legt einen großen Wert auf gut platzierte Publikationen und ein hoher Anteil der Publikationen entstammten internationale Kooperationen, eines der Hauptgründe für die zweite Platzierung im Ranking der internationalsten Universitäten (THE 2016). Um die Reichweite und Zugang zu erhöhen, setzt die Universität auf die Online-Plattform orbi.lu (orbilu .uni.lu) - ein Online Repository, in dem alle Publikationen der Universitätsmitglieder verzeichnet und zumindest alle Beiträge in peer-reviewed Journalen auf Anfrage oder direkt verfügbar sind. Am 1. Februar 2016 sind 2305 Publikationen im Bereich Bildung und Unterricht ausgewiesen. Pro Jahr sind das zwischen 150 und 200 Publikationen im erziehungswissenschaftlichen Bereich (2014: 202, 2015: 147; Stichtag: 1. Februar 2016). Diese Zahlen unterschätzen die tatsächliche Zahl von Publikationen mit erziehungswissenschaftlichem und Bildungsbezug an der Universität Luxemburg massiv, da viele dieser Publikationen im Repository auch unter anderen Disziplinen (z.B. Geschichtswissenschaft, Politik, Sozialwissenschaften) verzeichnet sind. Werden alle Publikationen von Mitarbeitenden der Universität Luxemburg, die mit den Schlüsselworten „education“, „enseignement“ oder „Bildung" versehen sind, gelistet, steigt die Anzahl der Einträge mit Bildungsbezug auf 9888 .

Wie in Abbildung 1 ersichtlich wird, beinhalten die Publikationsstrategien ein breites Spektrum, wobei die Beiträge in meist peer-reviewten wissenschaftlichen Zeitschriften und die Beiträge in Sammelbänden den Schwerpunkt bilden. 
Abbildung 1: Publikationen in der Kategorie Bildung \& Unterricht in Prozent (Februar 2016; N $=2305$ )

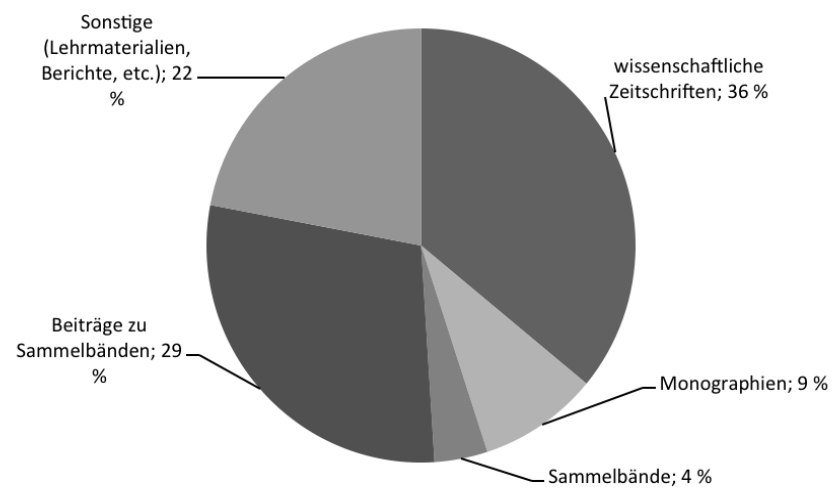

Quelle: Publikationsdatenbank der Universität Luxemburg, orbi.lu (Stichtag: 31. Januar 2016)

\section{Personal}

In der Fakultät FLSHASE - Faculty of Language and Literature, Humanities, Arts and Education - war im Dezember 201539 Prozent des Personals explizit dem Bereich der Bildung zugeordnet. Damit kommt den Erziehungsund Bildungswissenschaften innerhalb der Fakultät eine besondere Bedeutung zu. Auch wenn die fachlichen Hintergründe heterogen sind, eint die Mitarbeitenden dieses multidiziplinären Bereichs der Bildungsbezug. Zudem beschäftigen sich aber auch Mitarbeitende an anderen Bereichen mit Bildungsfragen (z.B. Educational Governance, Verschränkung von Sozialarbeit und Bildung), die hier nicht mit eingeschlossen sind.

In Abbildung 2 ist das Personal im Bereich Bildung, das explizit den Erziehungs- bzw. Bildungswissenschaften zugeordnet ist, verzeichnet. Wie bereits erwähnt, sind aufgrund der multidisziplinären Struktur der Universität weitere Erziehungswissenschaftlerinnen und Erziehungswissenschaftler in anderen Einheiten hier nicht berücksichtigt. Im Dezember 2015 verfügte der Bereich über sieben Ordinarien bzw. volle Professuren (im Hinblick auf die deutsche Besoldungsstruktur, W3-äquivalent), 14 Associate Professors (W2äquivalent) und 17 Oberassistenzen (in vielen Fällen vergleichbar mit unbefristeten akademischen Ratsstellen). Dazu kommen 13 Postdoc-Stellen und 24 Doktoranden im Bildungsbereich. 
Abbildung 2: Personal im Bereich Bildung (Dezember 2015)

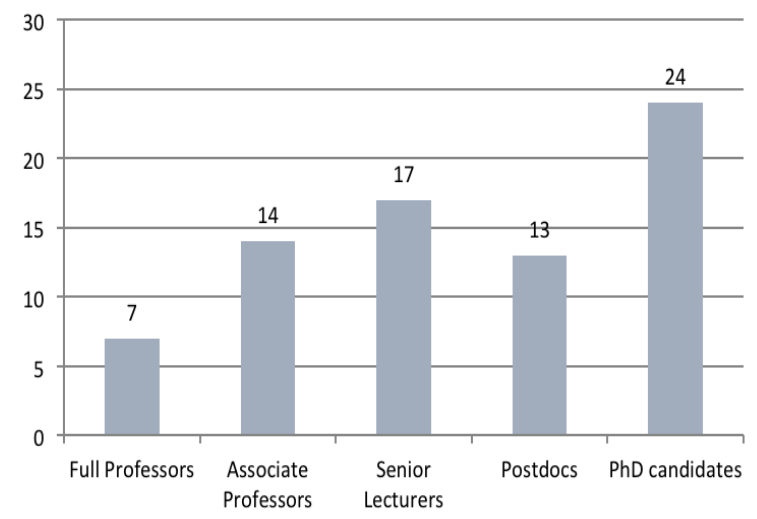

Quelle: Personalabteilung, Universität Luxemburg, Personalliste

Die Entwicklung des Bildungsbereichs seit 2006 ist in der Abbildung 3 dargestellt und zeigt die Zunahme des Personals, aber auch Stagnationen auf hohem Niveau.

Abbildung 3: Entwicklung des Personals im Bereich Bildung (2006 bis 2015)

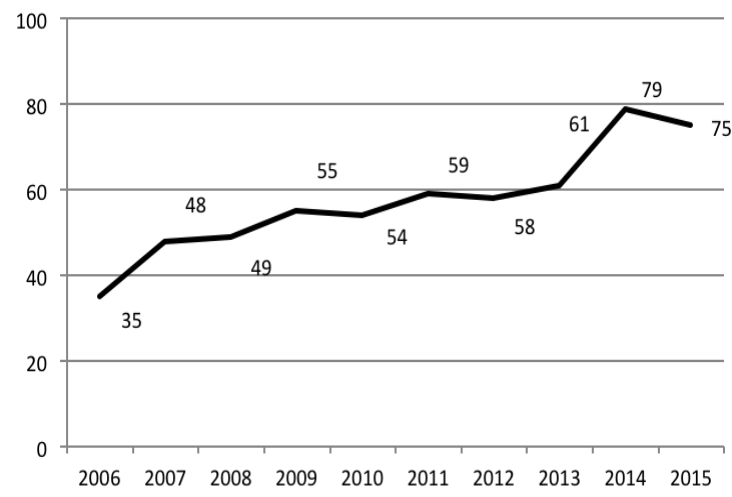

Quelle: Personalabteilung, Universität Luxemburg, Personalliste 


\section{Studiengänge}

Die Fakultät für Sprachwissenschaften und Literatur, Geisteswissenschaften, Kunst und Erziehungswissenschaften bietet akademische und professionelle (d.h. berufsorientierte) Studiengänge (Bachelor, Master, Weiterbildungszertifikate) sowie drei Doktorandenschulen (Erziehungswissenschaften, Sozialwissenschaften, Geisteswissenschaften) an. Die erziehungswissenschaftlichen Studiengänge werden im Folgenden kurz vorgestellt.

\subsection{Bachelorstudiengänge in Erziehungswissenschaft(en)}

Auf Bachelorstufe finden sich zwei berufsorientierte Studienprogramme mit erziehungswissenschaftlichem Bezug. Der vierjährige Bachelor en Sciences de l'Éducation vereint praktische und akademische erziehungswissenschaftliche Kompetenzen und Wissen. Dieser Studiengang (240 ECTS) richtet sich insbesondere an angehende Grundschullehrerinnen und Grundschullehrer, die im multilingualen und pluralen luxemburgischen Umfeld arbeiten werden. Unterrichtssprachen sind Französisch, Deutsch, Englisch und Lëtzebuergesch. Neben erziehungswissenschaftlichen Aspekten wie Bildungsgeschichte, Klassenführung, Kommunikation in der Schule und mit Eltern werden fachliche und fachdidaktische Kenntnisse vermittelt. Er entspricht somit einem Lehramtsstudiengang für Grundschulen, reicht aber darüber hinaus, weil auch andere Arbeitsfelder und weitergehende Studien (Master) nach dem entsprechenden Abschluss möglich sind. Der Bachelor en sciences sociales et éducatives, der in Deutsch, Französisch und Luxemburgisch vermittelt wird, hat einen sozial- und bildungswissenschaftlichen Charakter und richtet sich vor allem an angehende Sozialpädagoginnen, Sozialpädagogen, Sozialarbeiterinnen und Sozialarbeiter sowie Beratende und Betreuende im Sozialwesen. Der generalistische grundständige Studiengang bereitet einerseits auf weiterführende Masterstudiengänge und eine Karriere in Forschung und Wissenschaft vor. Andererseits können die Absolventen mit ihrem Abschluss in allen Praxisfeldern der sozialen und erzieherischen Arbeit in Luxemburg Fuß fassen. Das Studium (180 ECTS) beinhaltet neben Sozialarbeit/Sozialpädagogik, Forschungsmethoden, Projektmanagement auch starke bildungssoziologische und sozialpsychologische Studienanteile, etwa zu Bildungsungleichheiten. Unter den Studienzielen finden sich Aspekte wie die Analyse von sozialen Problemen (Arbeitslosigkeit, Gewalt, Delinquenz, Diskriminierung) oder die Anwendung ethischer Prinzipien (Menschenwürde, Respekt, Partizipation, Chancengleichheit).

\subsection{Masterstudiengänge in Erziehungswissenschaft(en)}

Erziehungswissenschaftliche Schwerpunkte finden sich in mindestens zwei akademischen Masterprogrammen und einem berufsorientierten Masterpro- 
gramm. Der englischsprachige akademische Master in Social Sciences and Educational Sciences (120 ECTS) findet sich an der Schnittstelle zwischen Sozialwissenschaften und Bildungs- bzw. Erziehungswissenschaft(en). Im Fokus des zweijährigen Studiums stehen Themen, die auf die Beziehung zwischen Bildung und Gesellschaft fokussieren und welche im Rahmen des Studiums forschungsorientiert behandelt werden. Dazu gehören der internationale Vergleich von Bildungs- und Sozialpolitiken, historische Analysen zur Entstehung von Bildungssystemen, institutionelle Regelungen in Bildungssystemen, das Ausmaß sozialer Ungleichheit in Bildungszertifikaten und Kompetenzen. Der ebenfalls akademische Master in Learning and Communication in Multilingual and Multicultural Contexts (120 ECTS) bietet englisch-, französisch- und deutschsprachige und multlinguale Lehrveranstaltungen an. Im Kern stehen hier Fragen der linguistischen und kulturellen Vielfalt in verschiedenen Feldern, wobei die Schule ein zentrales Feld darstellt. Die disziplinären Felder, die in diesem zweijährigen Studiengang vertreten sind, reichen von den Erziehungswissenschaft(en) über die Soziolinguistik, angewandte Linguistik, Soziologie bis hin zur Digital Literacy. Qualitative Forschungsmethoden sind ebenso Teil des Studienprogramms. Innerhalb der berufsorientierten bzw. berufsbegleitenden Studiengänge auf Masterniveau weist der Master Management und Coaching im Bildungs- und Sozialwesen (Sprachen: Deutsch und Französisch) einen starken erziehungswissenschaftlichen Anteil auf. Zur Zielgruppe gehören Fachkräfte mit Führungs- und Betreuungsaufgaben im Bildungs- und Sozialbereich. Aufgrund des berufsbegleitenden Charakters werden die 120 ECTS in drei Jahren absolviert. Die Studierenden müssen über mindestens drei Jahre Berufserfahrung verfügen. Das Programm ist international, denn die besondere Situation im Bildungsund Sozialwesen Luxemburgs wird im internationalen Vergleich herausgearbeitet, interdisziplinär, denn die Dozierenden vertreten erziehungswissenschaftliche, sozialwissenschaftliche oder psychologische Herangehensweisen, und praxisbezogen, denn Wissen und Erfahrungen aus den Arbeitsfeldern der Studierenden - dazu gehören u.a. Schulleitende, Lehrerinnen und Lehrer aus dem Grundschul- und Sekundarbereich, Mitarbeitende in der Jugendhilfe oder Kinderbetreuung - werden systematisch reflektiert und analysiert. Es gibt die zwei Studienprofile „Bildungswesen“ und „Sozialwesen“, wobei es neben profilübergreifenden Veranstaltungen wie Forschungsmethoden und Evaluation auch profilspezifische Veranstaltungen gibt. Die erziehungswissenschaftlichen Veranstaltungen des Bildungswesen-Profils vermitteln u.a. Wissen zu Bildungssystemen, Inklusion, pädagogischer Diagnostik, Lerntheorien und Interaktionen im Klassenzimmer. Schließlich gibt es im Umfeld des beschriebenen professionellen Masterprogramms auch ein Weiterbildungszertifikat (20 ECTS), das Zertifikat Tutoring und Mentoring im Bildungsbereich, welches angehenden und auch aktiven Tutorinnen und Tutoren im Verlaufe eines Jahres die nötigen Kompetenzen vermittelt. Zur 
Kernzielgruppe gehören Lehrerinnen und Lehrer in Grundschulen und Sekundarschulen, die als Tutoren Studierende während des praktischen Teils ihrer Lehrerinnen- bzw. Lehrerausbildung betreuen wollen. Eine starke Säule innerhalb des Studiums stellt die Reflexion der eigenen Unterrichtsentwicklung und der Praxis als Tutorin bzw. Tutor dar. Von der Gründung der Universität bis zum Jahr 2014 war auch die Luxemburgische Sekundarlehrerinnen- und Sekundarlehrerausbildung Formation pédagogique in die Fakultät der Universität Luxemburg integriert. Diese Aufgabe wurde von dem am Beginn angesprochenen Institut IFEN des Luxemburgischen Bildungsministeriums übernommen. Erste Schritte für einen universitären Masterstudiengang im Sekundarlehramt an der Universität Luxemburg sind allerdings bereits ge$\tan$ (vgl. Jovanovic/Priem 2012).

\subsection{Doktorandenausbildung in Erziehungswissenschaft(en)}

Doktoranden an der Universität Luxemburg haben insgesamt drei bis vier Jahre Zeit, ihr Projekt zu verfolgen und ihre Dissertation zu verfassen. Ein erfolgreicher Abschluss in dieser vergleichsweise ambitionierten Zeitplanung wird durch verschiedene Bedingungen möglich gemacht: Dem Doktoranden stehen von Beginn an neben dem Betreuenden (,Doktorvater" oder „Doktormutter") zwei weitere Betreuende zur Seite. In regelmäßigen Treffen wird der Fortschritt reflektiert und es werden Hinweise gegeben. Doktoranden in Haushalt- und Projektstellen an der Universität Luxemburg haben Vollzeitverträge (100 Prozent). Doktoranden auf Haushaltstellen arbeiten 80 Prozent an ihrer Doktorarbeit und werden nur zu höchstens 20 Prozent ihres Arbeitspensums mit anderen Aufgaben wie Lehre und Mitarbeit an anderen Publikationen betraut. Doktoranden in Projekten fokussieren zu 100 Prozent ihr Projekt.

Die Universität Luxemburg besitzt seit 2011 eine Besonderheit in der Ausbildung von Doktoranden im Bereich der Erziehungswissenschaften, eine Doctoral School in Educational Sciences. Dieses Studienangebot umfasst 20 ECTS und kann je nachdem in einem Jahr oder in zwei bis drei Jahren absolviert werden. In der Doktorandenausbildung sind verschiedene Disziplinen vertreten, die für die Bildungsforschung relevant sind. Dazu gehören Bereiche wie Bildungsgeschichte, Bildungssoziologie, Psychologie genauso wie Soziolinguistik. Zu den angebotenen Veranstaltungen gehören bereichsspezifische Doktorandenseminare, übergreifende Forschungskolloquien und Kurse, die allgemeine wissenschaftliche Fähigkeiten vermitteln (Schreib-, Kommunikations- oder Methodenkurse). Die spezifischen Veranstaltungen werden entlang von drei thematischen Achsen angeboten: a) History, Theory and Sociology of Education, b) Learning, Cognition and Development und c) Multilingualism in Education and Society. Die Doctoral School fördert für interessierte Studierende auch Auslandsaufenthalte, in den Vereinigten Staaten etwa an der Stanford University oder der University of Wisconsin-Madison sowie in Brasilien, Großbritannien oder Südtirol. Herz der Doctoral 
School ist das zweiwöchige multidisziplinäre Kolloquium in dem alle Promovierende im Verlauf ihres Projektes zwei Mal vorstellen.

\section{Studierende}

Die Studierendenzahlen der jungen Universität Luxemburg sind im Zuge der Einführung der verschiedenen Studiengänge insgesamt - in der Gesamtzahl der Studierenden in dieser Statistik Bachelor-, Master-, Doktorats- sowie Weiterbildungsstudiengänge, inklusive Nicht-Bologna-Studiengänge enthalten - kontinuierlich gestiegen, wobei sich für das letzte Berichtsjahr 2014/2015 eine Sättigung hinsichtlich einiger Studiengänge einstellt. Wie an anderen Universitäten auch, sind die Studierendenzahlen in den Bachelorstudiengänge höher als in den anderen Studiengängen (Abbildung 4).

Abbildung 4: Entwicklung der Studierendenzahlen der Universität Luxemburg vom Wintersemester 2005/2006 bis zum Wintersemester 2014/2015

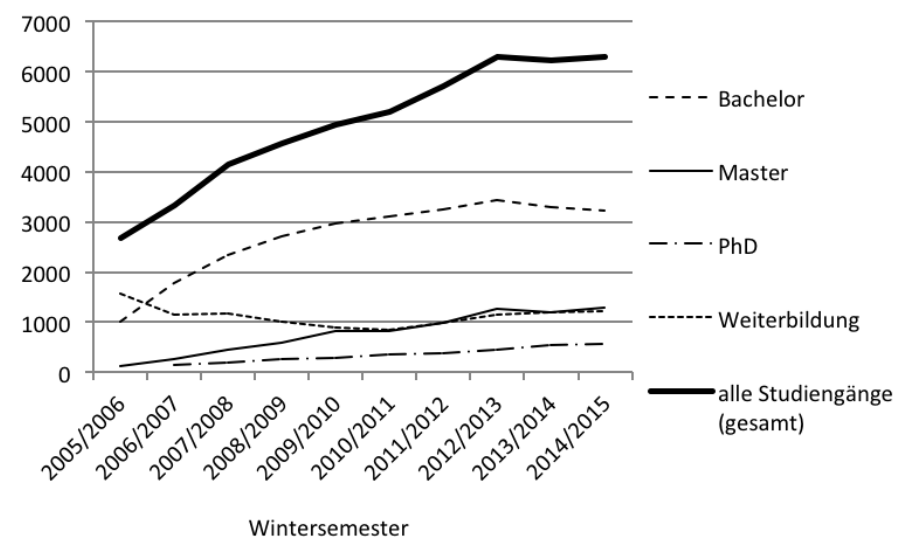

Quelle: Student Registration Statistics, Universität Luxemburg (Stichtag: 31. Dezember 2014)

Im akademischen Jahr 2014/2015 waren 516 Bachelorstudierende im Bereich Erziehungswissenschaft(en) und Lehrerinnen- und Lehrerbildung eingeschrieben, was 16 Prozent aller Bachelorstudierenden an der Universität Luxemburg im entsprechenden Zeitraum entspricht. In den erziehungswissenschaftlichen Masterstudiengängen waren 120 Studierende (9,4 Prozent aller Studierenden) eingeschrieben. Zum gleichen Zeitpunkt waren 37 Doktoranden im Bereich der Erziehungswissenschaften eingeschrieben (6,5 Prozent aller Doktoranden). Die erziehungswissenschaftlichen Weiterbildungsstudien- 
gänge besuchten im akademischen Jahr 2014/2015 285 Studierende; dies entspricht 23,7 Prozent aller Weiterbildungsstudierenden an der Universität Luxemburg.

Im Hinblick auf die erfolgreichen Absolventinnen und Absolventen sehen die Zahlen für das akademische Jahr 2013/2014 für den Bereich Erziehungswissenschaften und Lehrerinnen- und Lehrerbildung folgendermaßen aus: Die Bachelorprogramme haben 123 Studierende (19,6 Prozent aller Absolventinnen und Absolventen der Universität Luxemburg) erfolgreich abgeschlossen, die Masterprogramme hatten 38 erfolgreiche Absolventinnen und Absolventen (11,2 Prozent). Weil die Doktoratsprogramme erst kurz vorher eingeführt wurden und weil zudem einige Doktoranden aus den verschiedenen Bereichen der Erziehungswissenschaften in anderen Disziplinen ihre Doktorwürde erhielten (z.B. Geschichtswissenschaft, Psychologie, Soziologie), ist in der amtlichen Statistik der Universität nur eine abgeschlossene erziehungswissenschaftliche Promotion (1,2 Prozent aller abgeschlossenen Promotionen) für das akademische Jahr 2013/2014 verzeichnet. Dies unterschätzt allerdings die Zahl der erfolgreichen Promotionen im Bildungsbezug sehr stark. Die Weiterbildungsprogramme mit erziehungswissenschaftlichem Bezug kamen in dem Zeitraum auf 317 Absolventinnen und Absolventen, das entspricht 52,7 Prozent aller Absolventinnen und Absolventen der Universität Luxemburg.

Die hier berichteten Zahlen sind Mindestzahlen, denn sie berücksichtigen nur die explizit in den Erziehungswissenschaften zu verortenden Studierenden bzw. Absolventinnen und Absolventen. Einige sozialwissenschaftliche Studienprogramme mit bildungswissenschaftlicher Ausrichtung - dies betrifft auch einige der oben beschriebenen Studienprogramme - sind nicht in den Zahlen enthalten.

\section{Fazit}

Zusammenfassend kommt den Erziehungswissenschaft(en) an der Universität Luxemburg ein hoher Stellenwert zu. Sie zählt insbesondere zu den Grundpfeilern der human- und sozialwissenschaftlichen Fakultät FLSHASE. Aufgrund der multidisziplinären Strukturen innerhalb der Einheiten der Fakultät ist es andererseits schwierig „die Erziehungswissenschaft“ als Disziplin auszumachen, denn es finden sich häufig Verknüpfungen von Teilbereichen wie der Unterrichtsforschung, der Bildungssoziologie, der Bildungsgeschichte oder der Pädagogischen Psychologie. Was den Standort Luxemburg einzigartig und attraktiv macht, sind seine Internationalität, seine multidisziplinäre Ausrichtung, seine Ressourcenausstattung und seine Multilingualität. Dies kommt Forschung und Lehre, Mitarbeitenden und Studierenden zugute. Auch wenn die angesprochene Multidisziplinarität aus den traditionellen disziplinä- 
ren Traditionen heraus schwierig und aus dieser Perspektive für die Universität Luxemburg mehr disziplinäre Zuordnung wünschenswert erscheint, bietet dieses Beispiel aber dennoch auch Anregungen für die aktuellen Diskurse um die Erziehungswissenschaft(en): Inwieweit ist es notwendig (und ist es überhaupt möglich), für die Erziehungswissenschaft einen engen disziplinären Kern zu definieren, oder erfordert die Erforschung von Gegenständen rund um Bildung und Erziehung nicht gerade die Mehrzahl des Begriffs, d.h. Erziehungswissenschaften?

Andreas Hadjar, Prof. Dr., ist Hochschullehrer für Sociology of Education an der Universität Luxemburg.

\section{Literatur}

Jovanovic, Victor/Priem, Karin (2012): Multikulturalität und Mehrsprachigkeit. Lehrerinnen- und Lehrerbildung in Luxemburg. In: RangoschSchneck, E. (Hrsg.): Lehrer Lernen Migration: Außen- und Innenperspektiven einer, ,interkulturellen Lehrerbildung“. Hohengehren: Schneider Verlag, S. 83-98.

Meyer, Morgan B. (2008): The dynamics of science in a small Country: The case of Luxembourg. In: Science and Public Policy 35, 5, S. 361-371.

Powell, Justin J. W. (2015): Hochschulbildung und Forschung in Luxemburg. Bildungsbericht Luxemburg 2015. Luxembourg: MENJE/University of Luxembourg, S. 109-118.

THE-Times Higher Education (2016): The world's most international universities 2016. London: THE. www.timeshighereducation.com/features/200most-international-universities-world-2016 [Zugriff: 22. April 2016].

Universität Luxemburg (2016): Kennzahlen. Esch-Belval: Universität Luxemburg. http://wwwde.uni.lu/universitaet/ueber_die_universitaet/kennz ahlen [Zugriff: 14. Februar 2016]. 